\title{
Potentially Preventable Deaths Among the Five Leading Causes of Death - United States, 2010 and 2014
}

\begin{abstract}
Macarena C. García, DrPH${ }^{1}$; Brigham Bastian ${ }^{2}$; Lauren M. Rossen, $\mathrm{PhD}^{2}$; Robert Anderson, $\mathrm{PhD}^{2}$; Arialdi Miniño, $\mathrm{MPH}^{2}$; Paula W. Yoon, ScD ${ }^{1}$; Mark Faul, $\mathrm{PhD}^{3}$; Greta Massetti, $\mathrm{PhD}^{4}$; Cheryll C. Thomas, $\mathrm{MSPH}^{4}$; Yuling Hong, $\mathrm{MD}^{4}$; Michael F. Iademarco, $\mathrm{MD}^{1}$
\end{abstract}

Death rates by specific causes vary across the 50 states and the District of Columbia.* Information on differences in rates for the leading causes of death among states might help state health officials determine prevention goals, priorities, and strategies. CDC analyzed National Vital Statistics System data to provide national and state-specific estimates of potentially preventable deaths among the five leading causes of death in 2014 and compared these estimates with estimates previously published for 2010. Compared with 2010, the estimated number of potentially preventable deaths changed (supplemental material at https://stacks.cdc.gov/view/cdc/42472); cancer deaths decreased 25\% (from 84,443 to 63,209), stroke deaths decreased $11 \%$ (from 16,973 to 15,175), heart disease deaths decreased 4\% (from 91,757 to 87,950), chronic lower respiratory disease (CLRD) (e.g., asthma, bronchitis, and emphysema) deaths increased 1\% (from 28,831 to 29,232), and deaths from unintentional injuries increased 23\% (from 36,836 to 45,331 ). A better understanding of progress made in reducing potentially preventable deaths in the United States might inform state and regional efforts targeting the prevention of premature deaths from the five leading causes in the United States.

To determine significant changes in the number of potentially preventable deaths for the five leading causes of death in the United States, CDC analyzed National Vital Statistics System mortality data from 2014 (1) using the same analytic model presented in the original report that used 2010 data as benchmarks (2). The number of potentially preventable deaths per year per state in persons aged $<80$ years was determined by comparing the number of expected deaths (based on the cause-specific average death rate of the three states with the lowest 2008-2010

\footnotetext{
*http://www.cdc.gov/nchs/data/nvsr/nvsr61/nvsr61_04.pdf.
}

average rate by age groups) with the number of observed deaths in 2010 and 2014. Further detail on age-adjusted rates by state and cause can be found in yearly publications on final death data (1).

Population estimates for 2010 and 2014 were produced by the U.S. Census Bureau. The calculations of potentially preventable deaths were restricted to U.S. residents and deaths in persons aged $<80$ years. Premature death was defined as a death that occurred in a person aged $<80$ years, based on the average life expectancy for the total U.S. population, which was nearly

\section{INSIDE}

1256 Trends in Obesity Among Participants Aged 2-4 Years in the Special Supplemental Nutrition Program for Women, Infants, and Children United States, 2000-2014

1261 CDC Grand Rounds: A Public Health Approach to Detect and Control Hypertension

1265 Disparities in the Prevalence of Diagnosed Diabetes _ United States, 1999-2002 and 2011-2014

1270 Global Routine Vaccination Coverage, 2015

1274 Notes from the Field: Fungal Bloodstream Infections Associated with a Compounded Intravenous Medication at an Outpatient Oncology Clinic - New York City, 2016

1276 Notes from the Field: Cardiac Dysrhythmias After Loperamide Abuse — New York, 2008-2016

1278 Announcements

1281 QuickStats

Continuing Education examination available at http://www.cdc.gov/mmwr/cme/conted_info.html\#weekly.

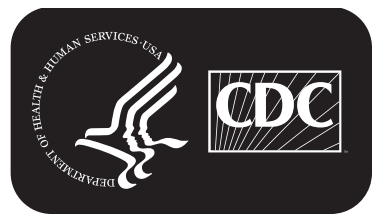

U.S. Department of Health and Human Services Centers for Disease Control and Prevention 
79 years in 2010 (2). Analysis was restricted to deaths with an underlying cause of death among the five leading causes, based on International Classification of Diseases, 10th Revision (ICD-10) codes. ${ }^{\dagger}$ The five leading causes of death represented $63 \%$ of all deaths in 2014 , a decrease of $2.3 \%$ compared with 2010. In 2014 the next five most frequent causes accounted for approximately $12 \%$ of deaths (3).

The number of potentially preventable deaths for each of the five leading causes of death by state in 2014 was calculated in four steps. The first step was to calculate and rank state disease-specific death rates by age group. Using 2008-2010 data, the three states with the lowest observed death rates for each age group and specific cause of death category were selected and their death rates averaged across the three states to calculate a lowest average age-specific death rate for each cause of death. For example, during 2008-10, among persons aged 40-49 years, the three states with the lowest rate of death from unintentional injuries were Maryland, New Jersey, and New York, and the benchmark average was 25.2 (supplemental material at https://stacks. cdc.gov/view/cdc/42342).

The average of the lowest three states was chosen to minimize the effect of any extreme outlier and to represent the low end of the distribution of death rates among the states. The second

$\dagger$ Diseases of the heart codes I00-I09, I1 1, I13, I20-I51; cancer C00-C97; CLRD J40-J47; cerebrovascular diseases (stroke) I60-I69; and unintentional injuries V01-X59, Y85-Y86. step was to calculate expected deaths for each age group and state by multiplying the age-specific state populations for 2010 by the 2010 benchmark death rates (i.e., the lowest three-state average age-specific death rates for each cause). Total expected deaths for each cause and state were calculated by summing expected deaths over all age groups aged $<80$ years, effectively taking into account differences in mortality across age groups. These state-specific and cause-specific expected death counts represent the number of deaths expected if all states were to achieve the 2010 death rate benchmarks (2). Third, the 2010 potentially preventable deaths were calculated by subtracting expected deaths from 2010 observed deaths. Finally, the same 2010 benchmark death rates for each cause were used to calculate 2014 potentially preventable deaths by repeating the third and fourth steps with 2014 population and mortality data. Specifically, the number of expected deaths in 2014 was calculated by multiplying the 2010 benchmark death rates by the 2014 age-specific populations; these expected counts were then subtracted from 2014 observed deaths. The numbers of potentially preventable deaths for each cause were assumed to follow a Poisson distribution, and standard errors were calculated, taking into account stochastic variation, consistent with methods described previously (2), in both the expected and observed number of deaths $\$$ for each cause and year. Statistically significant changes from 2010-2014 were

\$Standard error $=$ the square root of [expected number + observed number of deaths].

The MMWR series of publications is published by the Center for Surveillance, Epidemiology, and Laboratory Services, Centers for Disease Control and Prevention (CDC), U.S. Department of Health and Human Services, Atlanta, GA 30329-4027.

Suggested citation: [Author names; first three, then et al., if more than six.] [Report title]. MMWR Morb Mortal Wkly Rep 2016;65:[inclusive page numbers].

\section{Centers for Disease Control and Prevention}

Thomas R. Frieden, MD, MPH, Director

Harold W. Jaffe, MD, MA, Associate Director for Science

Joanne Cono, MD, ScM, Director, Office of Science Quality

Chesley L. Richards, MD, MPH, Deputy Director for Public Health Scientific Services

Michael F. Iademarco, MD, MPH, Director, Center for Surveillance, Epidemiology, and Laboratory Services

\section{MMWR Editorial and Production Staff (Weekly)}

Sonja A. Rasmussen, MD, MS, Editor-in-Chief

Charlotte K. Kent, PhD, MPH, Executive Editor Jacqueline Gindler, MD, Editor

Teresa F. Rutledge, Managing Editor

Douglas W. Weatherwax, Lead Technical Writer-Editor

Stacy A. Benton, Soumya Dunworth, PhD, Teresa M. Hood, MS, Technical Writer-Editors

Timothy F. Jones, MD, Chairman

Matthew L. Boulton, MD, MPH

Virginia A. Caine, MD

Katherine Lyon Daniel, PhD

Jonathan E. Fielding, MD, MPH, MBA

David W. Fleming, MD

MMWR Editorial Board

William E. Halperin, MD, DrPH, MPH

King K. Holmes, MD, PhD

Robin Ikeda, MD, MPH

Rima F. Khabbaz, MD

Phyllis Meadows, PhD, MSN, RN

Jewel Mullen, MD, MPH, MPA
Martha F. Boyd, Lead Visual Information Specialist

Maureen A. Leahy, Julia C. Martinroe,

Stephen R. Spriggs, Moua Yang, Tong Yang, Visual Information Specialists

Quang M. Doan, MBA, Phyllis H. King, Terraye M. Starr, Information Technology Specialists

and

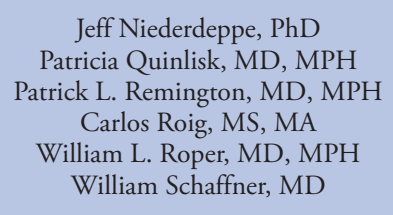

Jeff Niederdeppe, $\mathrm{PhD}$

trick L. Remington, MD, MPH

William L. Roper, MD, MPH

William Schaffner, MD 
assessed using a two-sided $\mathrm{z}$-test $(\mathrm{p}<0.01)$. Results are presented for the United States as a whole, by state, and by the 10 U.S. Department of Health and Human Services regions. 9

The five leading causes of death for persons aged $<80$ years in 2014 (diseases of the heart, malignancies [cancer], cerebrovascular diseases [stroke], chronic lower respiratory diseases [CLRD], and unintentional injuries [accidents]) represent 63\% of deaths from all causes. The estimated number of potentially preventable deaths and the proportion preventable among the five leading causes of death in persons aged $<80$ years were 87,950 for diseases of the heart (30\% preventable); 63,209 for cancer (15\% preventable); 45,331 for unintentional injuries

\footnotetext{
Region 1: Connecticut, Maine, Massachusetts, New Hampshire, Rhode Island, and Vermont. Region 2: New Jersey, New York, Puerto Rico, and the U.S. Virgin Islands. Region 3: Delaware, the District of Columbia, Maryland, Pennsylvania, Virginia, and West Virginia. Region 4: Alabama, Florida, Georgia, Kentucky, Mississippi, North Carolina, South Carolina, and Tennessee. Region 5: Illinois, Indiana, Michigan, Minnesota, Ohio, and Wisconsin. Region 6: Arkansas, Louisiana, New Mexico, Oklahoma, and Texas. Region 7: Iowa, Kansas, Missouri, and Nebraska. Region 8: Colorado, Montana, North Dakota, South Dakota, Utah, and Wyoming. Region 9: Arizona, California, Hawaii, Nevada, American Samoa, Commonwealth of the Northern Mariana Islands, Federated States of Micronesia, Guam, Marshall Islands, and Republic of Palau. Region 10: Alaska, Idaho, Oregon, and Washington.
}

(43\% preventable); 29,232 for CLRD (36\% preventable); and 15,175 for stroke (28\% preventable) (Figure).

Potentially preventable deaths from cancer declined $25 \%$ from 2010 to 2014 (the increase in the expected number of deaths was greater than the increase in the observed number). This decline appears to be driven by a $12 \%$ decrease in the age-adjusted death rate from lung cancer from 2010 and 2014. Decreases in age-adjusted death rates from cancer were observed across all U.S. states, except the District of Columbia (supplemental material at https://stacks.cdc.gov/ view/cdc/42343). The expected number of deaths was based on benchmark death rates from 2010; however, cancer-related death rates declined during 2010-2014. In both 2010 and 2014 the Southeast (Region 4) had the highest number of potentially preventable deaths for each of the five leading causes of death (Table 1). In 2014, the Northwest (Region 10) had the lowest number of potentially preventable deaths for each of the five leading causes of death except deaths from CLRD and unintentional injuries, where the lowest number occurred in New York and New Jersey (Region 2) (Table 2).

Consistent with increases in population since 2010, particularly among older age groups, the number of observed deaths

FIGURE. Number of potentially preventable deaths among the five leading causes of death, for persons aged $<80$ years — United States, 2010 and 2014

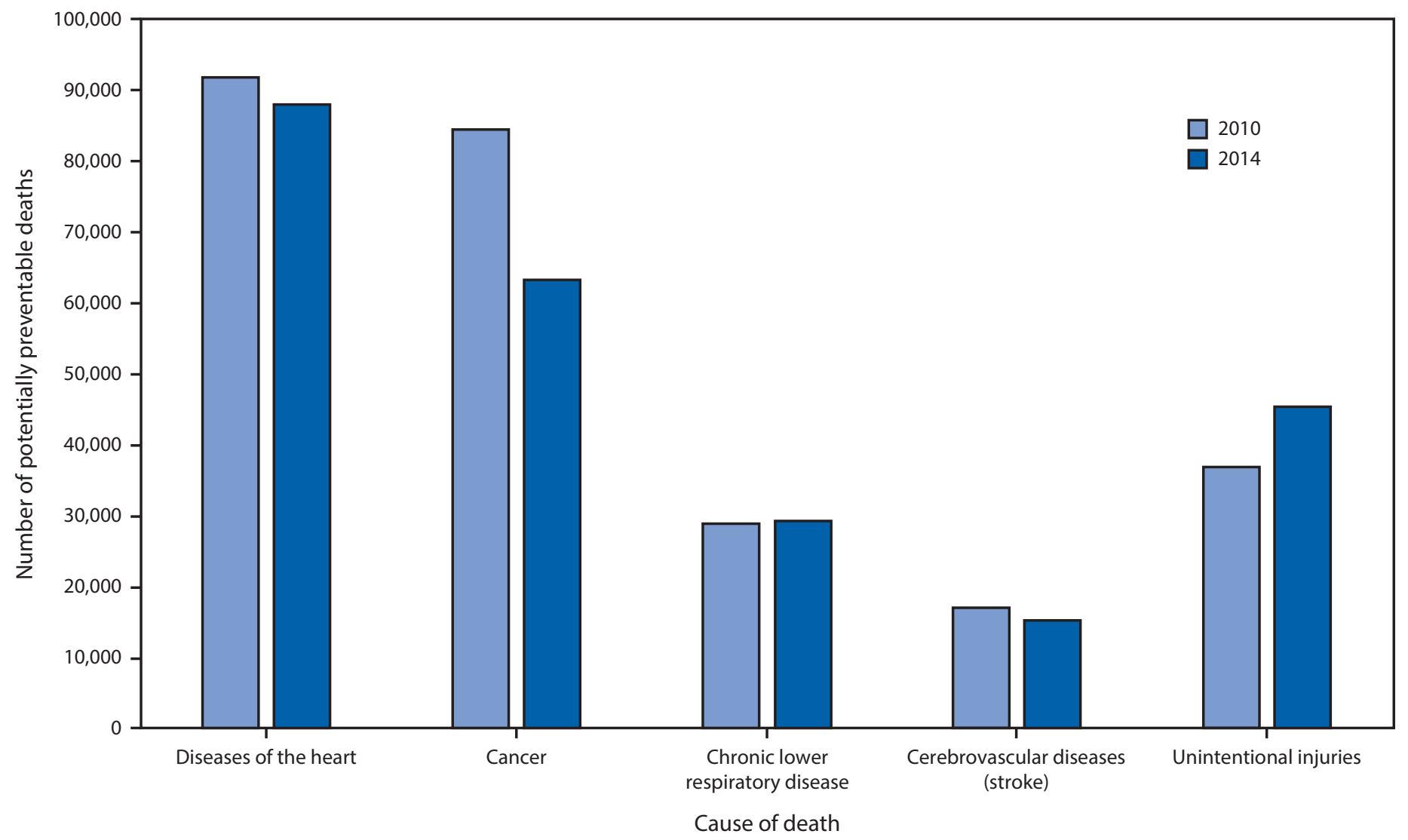


TABLE 1. Number of expected, observed, and potentially preventable deaths among the five leading causes of death and significant changes in potentially preventable deaths, for persons aged $<80$ years, by U.S. Department of Health and Human Services (HHS) region United States, 2010 and 2014

\begin{tabular}{|c|c|c|c|c|c|c|c|}
\hline \multirow[b]{2}{*}{ HHS region } & \multicolumn{3}{|c|}{2010} & \multicolumn{3}{|c|}{2014} & \multirow[b]{2}{*}{$\begin{array}{c}\text { Z-test } \\
\text { significance }\end{array}$} \\
\hline & Expected & Observed & $\begin{array}{l}\text { Potentially } \\
\text { preventable }\end{array}$ & Expected & Observed & $\begin{array}{c}\text { Potentially } \\
\text { preventable }\end{array}$ & \\
\hline \multicolumn{8}{|c|}{ Diseases of the heart } \\
\hline 1 & 8,904 & 10,286 & 1,382 & 9,798 & 10,468 & 670 & * \\
\hline 2 & 16,765 & 24,477 & 7,712 & 18,170 & 24,272 & 6,102 & * \\
\hline 3 & 18,327 & 28,563 & 10,236 & 20,099 & 29,185 & 9,086 & * \\
\hline 4 & 38,367 & 65,198 & 26,831 & 43,235 & 69,897 & 26,662 & \\
\hline 5 & 30,726 & 47,280 & 16,554 & 33,618 & 50,437 & 16,819 & \\
\hline 6 & 20,656 & 35,898 & 15,242 & 23,245 & 39,907 & 16,662 & * \\
\hline 7 & 8,281 & 12,769 & 4,488 & 8,958 & 13,425 & 4,467 & \\
\hline 8 & 5,782 & 6,464 & 682 & 6,616 & 7,325 & 709 & \\
\hline 9 & 26,030 & 33,352 & 7,322 & 29,622 & 35,133 & 5,511 & * \\
\hline 10 & 7,422 & 8,401 & 979 & 8,539 & 9,216 & 677 & \\
\hline \multicolumn{8}{|c|}{ Malignant neoplasms (cancers) } \\
\hline 1 & 15,587 & 19,061 & 3,474 & 17,216 & 18,995 & 1,779 & * \\
\hline 2 & 29,259 & 34,735 & 5,476 & 31,827 & 34,826 & 2,999 & * \\
\hline 3 & 32,039 & 42,003 & 9,964 & 35,241 & 43,236 & 7,995 & * \\
\hline 4 & 66,962 & 90,439 & 23,477 & 75,522 & 95,461 & 19,939 & * \\
\hline 5 & 53,686 & 71,553 & 17,867 & 58,975 & 73,529 & 14,554 & * \\
\hline 6 & 36,074 & 46,950 & 10,876 & 40,693 & 49,216 & 8,523 & * \\
\hline 7 & 14,443 & 19,028 & 4,585 & 15,692 & 19,653 & 3,961 & \\
\hline 8 & 10,123 & 10,708 & 585 & 11,625 & 11,387 & $-238^{\dagger}$ & * \\
\hline 9 & 45,439 & 50,611 & 5,172 & 51,835 & 53,179 & 1,344 & * \\
\hline 10 & 13,041 & 15,861 & 2,820 & 15,018 & 16,700 & 1,682 & * \\
\hline \multicolumn{8}{|c|}{ Cerebrovascular diseases (stroke) } \\
\hline 1 & 1,722 & 1,863 & 141 & 1,914 & 1,781 & -133 & * \\
\hline 2 & 3,261 & 3,742 & 481 & 3,556 & 3,716 & 160 & * \\
\hline 3 & 3,568 & 5,239 & 1,671 & 3,947 & 5,511 & 1,564 & \\
\hline 4 & 7,538 & 12,960 & 5,422 & 8,567 & 13,934 & 5,367 & \\
\hline 5 & 5,988 & 8,832 & 2,844 & 6,603 & 9,143 & 2,540 & \\
\hline 6 & 4,040 & 7,174 & 3,134 & 4,575 & 7,749 & 3,174 & \\
\hline 7 & 1,628 & 2,405 & 777 & 1,773 & 2,490 & 717 & \\
\hline 8 & 1,128 & 1,374 & 246 & 1,302 & 1,440 & 138 & \\
\hline 9 & 5,078 & 6,904 & 1,826 & 5,822 & 6,952 & 1,130 & * \\
\hline 10 & 1,439 & 1,867 & 428 & 1,679 & 1,991 & 312 & \\
\hline \multicolumn{8}{|c|}{ Chronic lower respiratory diseases (CLRD) } \\
\hline 1 & 2,234 & 2,774 & 540 & 2,505 & 3,068 & 563 & \\
\hline 2 & 4,218 & 4,794 & 576 & 4,634 & 4,697 & 63 & * \\
\hline 3 & 4,630 & 6,951 & 2,321 & 5,166 & 7,234 & 2,068 & \\
\hline 4 & 9,820 & 18,612 & 8,792 & 11,254 & 21,025 & 9,771 & * \\
\hline 5 & 7,740 & 13,494 & 5,754 & 8,623 & 14,669 & 6,046 & \\
\hline 6 & 5,174 & 9,539 & 4,365 & 5,911 & 10,547 & 4,636 & \\
\hline 7 & 2,111 & 4,318 & 2,207 & 2,317 & 4,644 & 2,327 & \\
\hline 8 & 1,442 & 2,447 & 1,005 & 1,686 & 2,681 & 995 & \\
\hline 9 & 6,514 & 8,447 & 1,933 & 7,550 & 8,977 & 1,427 & * \\
\hline 10 & 1,857 & 3,082 & 1,225 & 2,195 & 3,357 & 1,162 & \\
\hline \multicolumn{8}{|c|}{ Unintentional injuries (accidents) } \\
\hline 1 & 2,771 & 3,703 & 932 & 2,866 & 4,817 & 1,951 & * \\
\hline 2 & 5,357 & 5,692 & 335 & 5,531 & 6,824 & 1,293 & * \\
\hline 3 & 5,703 & 8,769 & 3,066 & 5,916 & 10,261 & 4,345 & * \\
\hline 4 & 11,650 & 23,804 & 12,154 & 12,338 & 24,789 & 12,451 & \\
\hline 5 & 9,724 & 15,104 & 5,380 & 9,984 & 17,898 & 7,914 & * \\
\hline 6 & 7,040 & 13,487 & 6,447 & 7,530 & 14,598 & 7,068 & * \\
\hline 7 & 2,566 & 4,720 & 2,154 & 2,639 & 4,901 & 2,262 & \\
\hline 8 & 1,985 & 3,479 & 1,494 & 2,136 & 4,046 & 1,910 & * \\
\hline 9 & 8,845 & 12,264 & 3,419 & 9,420 & 13,768 & 4,348 & * \\
\hline 10 & 2,414 & 3,840 & 1,426 & 2,569 & 4,358 & 1,789 & * \\
\hline
\end{tabular}

* Significant change from 2010 to $2014, \mathrm{p}<0.01$.

${ }^{\dagger}$ Negative potentially preventable deaths occurred when an HHS region included one or more of the states with the lowest three death rates (the lowest three death rates were averaged to create the benchmark death rates) for at least a few age groups. Negative potentially preventable deaths are preserved in this table to test changes from 2010 to 2014. 
TABLE 2. Number of expected, observed, and potentially preventable deaths among the five leading causes of death and significant changes in potentially preventable deaths, for persons aged <80 years, by state — United States, 2010 and 2014

\begin{tabular}{|c|c|c|c|c|c|c|c|}
\hline \multirow[b]{2}{*}{ State } & \multicolumn{3}{|r|}{2010} & \multicolumn{3}{|r|}{2014} & \multirow{2}{*}{$\begin{array}{c}\text { Z-test } \\
\text { significance }\end{array}$} \\
\hline & Expected & Observed & Potentially preventable $(95 \% \mathrm{Cl})$ & Expected & Observed & Potentially preventable $(95 \% \mathrm{Cl})$ & \\
\hline \multicolumn{8}{|l|}{ Diseases of the heart } \\
\hline Alabama & 2,993 & 6,604 & $3,611(3,419-3,803)$ & 3,266 & 6,933 & $3,667(3,469-3,865)$ & \\
\hline Alaska & 331 & 463 & $132(77-187)$ & 377 & 497 & $120(62-178)$ & \\
\hline Arizona & 3,885 & 4,735 & $850(668-1,032)$ & 4,512 & 5,061 & $549(357-741)$ & \\
\hline Arkansas & 1,845 & 3,808 & $1,963(1,816-2,110)$ & 1,998 & 4,258 & $2,260(2,105-2,415)$ & * \\
\hline California & 19,742 & 24,707 & $4,965(4,552-5,378)$ & 22,358 & 25,338 & $2,980(2,552-3,408)$ & * \\
\hline Colorado & 2,707 & 2,815 & $108(-38-254)$ & 3,153 & 3,246 & $93(-64-250)$ & \\
\hline Connecticut & 2,176 & 2,569 & $393(258-528)$ & 2,362 & 2,552 & $190(53-327)$ & \\
\hline Delaware & 575 & 857 & $282(208-356)$ & 658 & 929 & $271(193-349)$ & \\
\hline District of Columbia & 310 & 729 & $419(356-482)$ & 337 & 733 & $396(332-460)$ & \\
\hline Florida & 13,352 & 17,586 & $4,234(3,889-4,579)$ & 15,121 & 19,121 & $4,000(3,637-4,363)$ & \\
\hline Georgia & 5,120 & 9,103 & $3,983(3,749-4,217)$ & 5,890 & 9,911 & $4,021(3,775-4,267)$ & \\
\hline Hawaii & 836 & 1,007 & $171(87-255)$ & 920 & 1,217 & $297(206-388)$ & \\
\hline Idaho & 883 & 1,080 & $197(110-284)$ & 1,025 & 1,240 & $215(122-308)$ & \\
\hline Illinois & 7,249 & 11,424 & $4,175(3,907-4,443)$ & 7,898 & 11,839 & $3,941(3,666-4,216)$ & \\
\hline Indiana & 3,783 & 6,421 & $2,638(2,440-2,836)$ & 4,145 & 6,779 & $2,634(2,429-2,839)$ & \\
\hline lowa & 1,892 & 2,716 & $824(691-957)$ & 2,032 & 2,622 & $590(456-724)$ & \\
\hline Kansas & 1,636 & 2,248 & $612(490-734)$ & 1,766 & 2,402 & $636(509-763)$ & \\
\hline Kentucky & 2,662 & 5,332 & $2,670(2,495-2,845)$ & 2,912 & 5,798 & $2,886(2,703-3,069)$ & \\
\hline Louisiana & 2,609 & 5,784 & $3,175(2,995-3,355)$ & 2,861 & 6,149 & $3,288(3,102-3,474)$ & \\
\hline Maine & 928 & 1,083 & $155(67-243)$ & 1,026 & 1,167 & $141(49-233)$ & \\
\hline Maryland & 3,303 & 5,321 & $2,018(1,836-2,200)$ & 3,701 & 5,476 & $1,775(1,587-1,963)$ & \\
\hline Massachusetts & 3,926 & 4,416 & $490(311-669)$ & 4,333 & 4,382 & $49(-134-232)$ & * \\
\hline Michigan & 6,056 & 10,327 & $4,271(4,020-4,522)$ & 6,646 & 11,461 & $4,815(4,551-5,079)$ & * \\
\hline Minnesota & 3,050 & 2,720 & $-330^{\dagger}(-479$ to -181$)$ & 3,414 & 2,951 & $-463(-619$ to -307$)$ & \\
\hline Mississippi & 1,750 & 4,183 & $2,433(2,282-2,584)$ & 1,903 & 4,428 & $2,525(2,369-2,681)$ & \\
\hline Missouri & 3,691 & 6,553 & $2,862(2,664-3,060)$ & 4,011 & 7,113 & $3,102(2,895-3,309)$ & \\
\hline Montana & 650 & 826 & $176(101-251)$ & 733 & 910 & $177(98-256)$ & \\
\hline Nebraska & 1,063 & 1,252 & $189(95-283)$ & 1,149 & 1,288 & $139(42-236)$ & \\
\hline Nevada & 1,566 & 2,903 & $1,337(1,206-1,468)$ & 1,832 & 3,517 & $1,685(1,542-1,828)$ & * \\
\hline New Hampshire & 828 & 916 & $88(6-170)$ & 931 & 976 & $45(-41-131)$ & \\
\hline New Jersey & 5,243 & 7,106 & $1,863(1,645-2,081)$ & 5,703 & 7,145 & $1,442(1,220-1,664)$ & * \\
\hline New Mexico & 1,253 & 1,510 & $257(154-360)$ & 1,382 & 1,642 & $260(152-368)$ & \\
\hline New York & 11,522 & 17,371 & $5,849(5,516-6,182)$ & 12,467 & 17,127 & $4,660(4,323-4,997)$ & * \\
\hline North Carolina & 5,679 & 9,021 & $3,342(3,104-3,580)$ & 6,456 & 9,223 & $2,767(2,522-3,012)$ & * \\
\hline North Dakota & 406 & 512 & $106(47-165)$ & 437 & 542 & $105(44-166)$ & \\
\hline Ohio & 7,164 & 11,875 & $4,711(4,441-4,981)$ & 7,736 & 12,697 & $4,961(4,681-5,241)$ & \\
\hline Oklahoma & 2,267 & 4,857 & $2,590(2,425-2,755)$ & 2,456 & 5,300 & $2,844(2,671-3,017)$ & \\
\hline Oregon & 2,364 & 2,421 & $58(-79-193)$ & 2,714 & 2,622 & $-92(-235-51)$ & \\
\hline Pennsylvania & 8,221 & 12,668 & $4,447(4,164-4,730)$ & 8,824 & 12,689 & $3,865(3,578-4,152)$ & * \\
\hline Rhode Island & 636 & 820 & $184(109-259)$ & 689 & 855 & $166(89-243)$ & \\
\hline South Carolina & 2,896 & 5,413 & $2,517(2,338-2,696)$ & 3,335 & 5,742 & $2,407(2,220-2,594)$ & \\
\hline South Dakota & 491 & 590 & $99(35-163)$ & 541 & 741 & $200(130-270)$ & \\
\hline Tennessee & 3,916 & 7,956 & $4,040(3,826-4,254)$ & 4,353 & 8,741 & $4,388(4,164-4,612)$ & \\
\hline Texas & 12,683 & 19,939 & $7,256(6,902-7,610)$ & 14,549 & 22,558 & $8,009(7,631-8,387)$ & * \\
\hline Utah & 1,194 & 1,229 & $35(-61-131)$ & 1,383 & 1,349 & $-34(-136-68)$ & \\
\hline Vermont & 411 & 482 & $71(12-130)$ & 457 & 536 & $79(17-141)$ & \\
\hline Virginia & 4,609 & 6,588 & $1,979(1,772-2,186)$ & 5,185 & 6,978 & $1,793(1,577-2,009)$ & \\
\hline Washington & 3,844 & 4,437 & $593(415-771)$ & 4,424 & 4,857 & $433(244-622)$ & \\
\hline West Virginia & 1,308 & 2,400 & 1,092 (973-1,211) & 1,395 & 2,380 & $985(865-1,105)$ & \\
\hline Wisconsin & 3,424 & 4,513 & $1,089(914-1,264)$ & 3,779 & 4,710 & $931(750-1,112)$ & \\
\hline Wyoming & 333 & 492 & $159(103-215)$ & 369 & 537 & $168(109-227)$ & \\
\hline United States & 181,261 & 272,688 & $91,757(90,436-93,078)$ & 201,902 & 289,265 & $87,950(86,576-89,324)$ & * \\
\hline \multicolumn{8}{|c|}{ Malignant neoplasms (cancers) } \\
\hline Alabama & 5,227 & 7,595 & $2,368(2,146-2,590)$ & 5,714 & 7,796 & $2,082(1,854-2,310)$ & \\
\hline Alaska & 588 & 703 & $115(45-185)$ & 670 & 782 & $112(37-187)$ & \\
\hline Arizona & 6,775 & 7,460 & 685 (451-919) & 7,857 & 8,085 & $228(-19-475)$ & * \\
\hline Arkansas & 3,219 & 4,720 & $1,501(1,326-1,676)$ & 3,487 & 4,897 & $1,410(1,231-1,589)$ & \\
\hline California & 34,454 & 38,226 & $3,772(3,244-4,300)$ & 39,157 & 39,678 & $521(-29-1071)$ & * \\
\hline Colorado & 4,752 & 4,944 & $192(-1-385)$ & 5,553 & 5,188 & $-365(-568$ to -162$)$ & * \\
\hline Connecticut & 3,805 & 4,367 & $562(385-739)$ & 4,144 & 4,219 & $75(-104-254)$ & * \\
\hline Delaware & 1,006 & 1,352 & $346(251-441)$ & 1,151 & 1,426 & $275(176-374)$ & \\
\hline
\end{tabular}

See table footnotes on page 1253. 
TABLE 2. (Continued) Number of expected, observed, and potentially preventable deaths among the five leading causes of death and significant changes in potentially preventable deaths, for persons aged <80 years, by state — United States, 2010 and 2014

\begin{tabular}{|c|c|c|c|c|c|c|c|}
\hline \multirow[b]{2}{*}{ State } & \multicolumn{3}{|r|}{2010} & \multicolumn{3}{|r|}{2014} & \multirow{2}{*}{$\begin{array}{c}\text { Z-test } \\
\text { significance }\end{array}$} \\
\hline & Expected & Observed & Potentially preventable $(95 \% \mathrm{Cl})$ & Expected & Observed & Potentially preventable $(95 \% \mathrm{CI})$ & \\
\hline District of Columbia & 543 & 742 & 199 (129-269) & 592 & 837 & $245(171-319)$ & \\
\hline Florida & 23,195 & 28,249 & $5,054(4,609-5,499)$ & 26,279 & 29,519 & $3,240(2,777-3,703)$ & * \\
\hline Georgia & 8,967 & 11,820 & $2,853(2,570-3,136)$ & 10,323 & 12,738 & $2,415(2,117-2,713)$ & \\
\hline Hawaii & 1,467 & 1,555 & $88(-20-196)$ & 1,616 & 1,693 & $77(-36-190)$ & \\
\hline Idaho & 1,546 & 1,753 & $207(94-320)$ & 1,796 & 1,995 & $199(78-320)$ & \\
\hline Illinois & 12,654 & 16,558 & $3,904(3,569-4,239)$ & 13,840 & 16,862 & $3,022(2,679-3,365)$ & * \\
\hline Indiana & 6,612 & 9,385 & $2,773(2,525-3,021)$ & 7,268 & 9,821 & $2,553(2,297-2,809)$ & \\
\hline lowa & 3,295 & 4,127 & $832(663-1,001)$ & 3,559 & 4,258 & $699(526-872)$ & \\
\hline Kansas & 2,854 & 3,624 & 770 (612-928) & 3,098 & 3,758 & $660(498-822)$ & \\
\hline Kentucky & 4,655 & 7,499 & $2,844(2,628-3,060)$ & 5,104 & 7,815 & $2,711(2,488-2,934)$ & \\
\hline Louisiana & 4,562 & 6,909 & $2,347(2,137-2,557)$ & 5,021 & 7,137 & $2,116(1,900-2,332)$ & \\
\hline Maine & 1,627 & 2,259 & $632(510-754)$ & 1,805 & 2,186 & $381(257-505)$ & * \\
\hline Maryland & 5,788 & 7,218 & $1,430(1,206-1,654)$ & 6,499 & 7,616 & $1,117(884-1,350)$ & \\
\hline Massachusetts & 6,865 & 8,319 & $1,454(1,212-1,696)$ & 7,609 & 8,341 & $732(484-980)$ & * \\
\hline Michigan & 10,600 & 14,394 & $3,794(3,484-4,104)$ & 11,671 & 14,884 & $3,213(2,894-3,532)$ & \\
\hline Minnesota & 5,328 & 6,273 & $945(734-1,156)$ & 5,992 & 6,267 & $275(58-492)$ & * \\
\hline Mississippi & 3,055 & 4,731 & $1,676(1,503-1,849)$ & 3,330 & 5,019 & $1,689(1,510-1,868)$ & \\
\hline Missouri & 6,442 & 9,023 & $2,581(2,337-2,825)$ & 7,020 & 9,345 & $2,325(2,074-2,576)$ & \\
\hline Montana & 1,143 & 1,304 & $161(64-258)$ & 1,291 & 1,445 & $154(51-257)$ & \\
\hline Nebraska & 1,852 & 2,254 & $402(276-528)$ & 2,014 & 2,292 & 278 (149-407) & \\
\hline Nevada & 2,743 & 3,370 & $627(474-780)$ & 3,205 & 3,723 & $518(355-681)$ & \\
\hline New Hampshire & 1,455 & 1,772 & $317(206-428)$ & 1,642 & 1,846 & $204(88-320)$ & \\
\hline New Jersey & 9,147 & 10,948 & $1,801(1,523-2,079)$ & 9,986 & 10,965 & $979(695-1,263)$ & * \\
\hline New Mexico & 2,194 & 2,393 & $199(66-332)$ & 2,423 & 2,451 & $28(-109-165)$ & \\
\hline New York & 20,112 & 23,787 & $3,675(3,264-4,086)$ & 21,842 & 23,861 & $2,019(1,600-2,438)$ & * \\
\hline North Carolina & 9,931 & 13,297 & $3,366(3,067-3,665)$ & 11,304 & 14,393 & $3,089(2,775-3,403)$ & \\
\hline North Dakota & 708 & 780 & $72(-4-148)$ & 767 & 852 & $85(6-164)$ & \\
\hline Ohio & 12,514 & 17,413 & $4,899(4,560-5,238)$ & 13,570 & 18,043 & $4,473(4,125-4,821)$ & \\
\hline Oklahoma & 3,957 & 5,787 & $1,830(1,637-2,023)$ & 4,293 & 5,896 & $1,603(1,405-1,801)$ & \\
\hline Oregon & 4,153 & 5,212 & 1,059 (869-1,249) & 4,771 & 5,496 & $725(526-924)$ & \\
\hline Pennsylvania & 14,340 & 19,114 & 4,774 (4416-5,132) & 15,463 & 19,064 & $3,601(3,237-3,965)$ & * \\
\hline Rhode Island & 1,112 & 1,423 & $311(212-410)$ & 1,210 & 1,442 & $232(131-333)$ & \\
\hline South Carolina & 5,079 & 7,063 & $1,984(1,768-2,200)$ & 5,846 & 7,487 & $1,641(1,415-1,867)$ & \\
\hline South Dakota & 856 & 1,054 & $198(112-284)$ & 950 & 1,115 & $165(76-254)$ & \\
\hline Tennessee & 6,853 & 10,185 & $3,332(3,076-3,588)$ & 7,622 & 10,694 & $3,072(2,807-3,337)$ & \\
\hline Texas & 22,143 & 27,141 & $4,998(4,563-5,433)$ & 25,469 & 28,835 & $3,366(2,909-3,823)$ & $*$ \\
\hline Utah & 2,080 & 1,931 & $-149(-273$ to -25$)$ & 2,413 & 2,105 & $-308(-440$ to -176$)$ & \\
\hline Vermont & 723 & 921 & $198(119-277)$ & 807 & 961 & $154(72-236)$ & \\
\hline Virginia & 8,073 & 10,162 & $2,089(1,824-2,354)$ & 9,090 & 10,651 & $1,561(1,286-1,836)$ & * \\
\hline Washington & 6,754 & 8,193 & $1,439(1,199-1,679)$ & 7,781 & 8,427 & $646(396-896)$ & * \\
\hline West Virginia & 2,289 & 3,415 & $1,126(978-1,274)$ & 2,446 & 3,642 & $1,196(1,043-1,349)$ & \\
\hline Wisconsin & 5,978 & 7,530 & $1,552(1,324-1,780)$ & 6,635 & 7,652 & 1,017 (783-1251) & * \\
\hline Wyoming & 585 & 695 & $110(40-180)$ & 651 & 682 & $31(-41-103)$ & \\
\hline United States & 316,652 & 400,949 & $84,443(82,783-86,103)$ & 353,645 & 416,182 & $63,209(61,489-64,929)$ & * \\
\hline \multicolumn{8}{|c|}{ Cerebrovascular diseases (stroke) } \\
\hline Alabama & 588 & 1,277 & $689(604-774)$ & 646 & 1,335 & $689(602-776)$ & \\
\hline Alaska & 62 & 91 & $29(5-53)$ & 72 & 87 & $15(-10-40)$ & \\
\hline Arizona & 771 & 848 & $77(-2-156)$ & 905 & 912 & $7(-77-91)$ & \\
\hline Arkansas & 365 & 718 & $353(289-417)$ & 398 & 738 & 340 (274-406) & \\
\hline California & 3,839 & 5,366 & $1,527(1,339-1,715)$ & 4,374 & 5,324 & $950(757-1,143)$ & * \\
\hline Colorado & 520 & 604 & $84(18-150)$ & 614 & 633 & $19(-50-88)$ & \\
\hline Connecticut & 420 & 425 & $5(-52-62)$ & 460 & 405 & $-55(-113-3)$ & \\
\hline Delaware & 113 & 170 & $57(24-90)$ & 130 & 172 & $42(8-76)$ & \\
\hline District of Columbia & 61 & 107 & $46(21-71)$ & 66 & 88 & $22(-2-46)$ & \\
\hline Florida & 2,655 & 3,481 & $826(672-980)$ & 3,030 & 3,812 & 782 (620-944) & \\
\hline Georgia & 989 & 1,965 & $976(869-1,083)$ & 1,150 & 2,060 & $910(799-1,021)$ & \\
\hline Hawaii & 163 & 244 & $81(41-121)$ & 182 & 234 & $52(12-92)$ & \\
\hline Idaho & 174 & 234 & $60(20-100)$ & 204 & 268 & $64(21-107)$ & \\
\hline Illinois & 1,412 & 2,047 & $635(520-750)$ & 1,547 & 2,175 & $628(508-748)$ & \\
\hline Indiana & 739 & 1,240 & $501(414-588)$ & 816 & 1,289 & $473(383-563)$ & \\
\hline lowa & 373 & 462 & $89(32-146)$ & 403 & 465 & $62(4-120)$ & \\
\hline Kansas & 321 & 485 & $164(108-220)$ & 349 & 489 & $140(83-197)$ & \\
\hline
\end{tabular}

See table footnotes on page 1253. 
TABLE 2. (Continued) Number of expected, observed, and potentially preventable deaths among the five leading causes of death and significant changes in potentially preventable deaths, for persons aged $<80$ years, by state - United States, 2010 and 2014

\begin{tabular}{|c|c|c|c|c|c|c|c|}
\hline \multirow[b]{2}{*}{ State } & \multicolumn{3}{|r|}{2010} & \multicolumn{3}{|r|}{2014} & \multirow{2}{*}{$\begin{array}{c}\text { Z-test } \\
\text { significance }\end{array}$} \\
\hline & Expected & Observed & Potentially preventable $(95 \% \mathrm{CI})$ & Expected & Observed & Potentially preventable $(95 \% \mathrm{Cl})$ & \\
\hline Kentucky & 520 & 934 & 414 (339-489) & 573 & 948 & $375(299-451)$ & \\
\hline Louisiana & 510 & 1,003 & $493(417-569)$ & 564 & 1,176 & $612(530-694)$ & \\
\hline Maine & 180 & 229 & $49(9-89)$ & 202 & 222 & $20(-20-60)$ & \\
\hline Maryland & 636 & 935 & $299(221-377)$ & 720 & 1,025 & $305(223-387)$ & \\
\hline Massachusetts & 761 & 807 & $46(-32-124)$ & 846 & 784 & $-62(-141-17)$ & \\
\hline Michigan & 1,178 & 1,743 & $565(459-671)$ & 1,306 & 1,792 & $486(377-595)$ & \\
\hline Minnesota & 592 & 662 & $70(1-139)$ & 669 & 705 & $36(-37-109)$ & \\
\hline Mississippi & 344 & 827 & $483(416-550)$ & 377 & 858 & $481(412-550)$ & \\
\hline Missouri & 724 & 1,164 & $440(355-525)$ & 793 & 1,263 & $470(381-559)$ & \\
\hline Montana & 127 & 162 & $35(2-68)$ & 146 & 182 & $36(1-71)$ & \\
\hline Nebraska & 209 & 294 & $85(41-129)$ & 227 & 273 & $46(2-90)$ & \\
\hline Nevada & 305 & 446 & 141 (87-195) & 361 & 482 & $121(64-178)$ & \\
\hline New Hampshire & 158 & 163 & $5(-30-40)$ & 181 & 174 & $-7(-44-30)$ & \\
\hline New Jersey & 1,015 & 1,319 & 304 (209-399) & 1,111 & 1,322 & $211(114-308)$ & \\
\hline New Mexico & 246 & 310 & $64(18-110)$ & 275 & 321 & $46(-2-94)$ & \\
\hline New York & 2,246 & 2,423 & $177(43-311)$ & 2,445 & 2,394 & $-51(-187-85)$ & \\
\hline North Carolina & 1,108 & 1,894 & $786(679-893)$ & 1,271 & 2,110 & $839(725-953)$ & \\
\hline North Dakota & 80 & 127 & $47(19-75)$ & 87 & 120 & $33(5-61)$ & \\
\hline Ohio & 1,400 & 2,271 & 871 (752-990) & 1,523 & 2,328 & $805(683-927)$ & \\
\hline Oklahoma & 448 & 889 & $441(369-513)$ & 488 & 894 & $406(333-479)$ & \\
\hline Oregon & 461 & 635 & 174 (109-239) & 536 & 699 & $163(94-232)$ & \\
\hline Pennsylvania & 1,611 & 2,194 & $583(462-704)$ & 1,740 & 2,388 & $648(522-774)$ & \\
\hline Rhode Island & 123 & 148 & $25(-7-57)$ & 135 & 114 & $-21(-52-10)$ & \\
\hline South Carolina & 567 & 1,119 & $552(472-632)$ & 661 & 1,185 & $524(440-608)$ & \\
\hline South Dakota & 97 & 126 & $29(0-58)$ & 107 & 108 & $1(-28-30)$ & \\
\hline Tennessee & 765 & 1,463 & $698(605-791)$ & 859 & 1,626 & $767(669-865)$ & \\
\hline Texas & 2,471 & 4,254 & $1,783(1,622-1,944)$ & 2,850 & 4,620 & $1,770(1,601-1,939)$ & \\
\hline Utah & 238 & 282 & $44(-1-89)$ & 276 & 325 & $49(1-97)$ & \\
\hline Vermont & 79 & 91 & $12(-14-38)$ & 90 & 82 & $-8(-34-18)$ & \\
\hline Virginia & 891 & 1,369 & $478(385-571)$ & 1,014 & 1,354 & $340(245-435)$ & \\
\hline Washington & 743 & 907 & 164 (84-244) & 867 & 937 & $70(-13-153)$ & \\
\hline West Virginia & 257 & 464 & $207(154-260)$ & 276 & 484 & $208(154-262)$ & \\
\hline Wisconsin & 667 & 869 & $202(125-279)$ & 742 & 854 & $112(34-190)$ & \\
\hline Wyoming & 65 & 73 & $8(-15-31)$ & 73 & 72 & $-1(-25-23)$ & \\
\hline United States & 35,390 & 52,360 & $16,973(16,392-17,554)$ & 39,737 & 54,707 & $15,175(14,573-15,777)$ & $*$ \\
\hline \multicolumn{8}{|c|}{ Chronic lower respiratory diseases } \\
\hline Alabama & 765 & 1,778 & $1,013(914-1,112)$ & 848 & 1,897 & $1,049(946-1,152)$ & \\
\hline Alaska & 77 & 112 & $35(8-62)$ & 92 & 116 & $24(-4-52)$ & \\
\hline Arizona & 1,004 & 1,558 & $554(455-653)$ & 1,189 & 1,870 & $681(573-789)$ & \\
\hline Arkansas & 476 & 1,101 & $625(547-703)$ & 523 & 1,339 & $816(731-901)$ & * \\
\hline California & 4,904 & 6,047 & $1,143(938-1,348)$ & 5,650 & 6,073 & $423(211-635)$ & * \\
\hline Colorado & 665 & 1,141 & $476(393-559)$ & 795 & 1,301 & $506(416-596)$ & \\
\hline Connecticut & 544 & 509 & $-35(-99-29)$ & 601 & 586 & $-15(-83-53)$ & \\
\hline Delaware & 147 & 224 & $77(39-115)$ & 172 & 231 & $59(20-98)$ & \\
\hline District of Columbia & 78 & 73 & $-5(-29-19)$ & 85 & 73 & $-12(-37-13)$ & \\
\hline Florida & 3,501 & 5,327 & $1,826(1,642-2,010)$ & 4,018 & 5,855 & $1,837(1,642-2,032)$ & \\
\hline Georgia & 1,263 & 2,413 & $1,150(1,031-1,269)$ & 1,486 & 2,729 & $1,243(1,116-1,370)$ & \\
\hline Hawaii & 212 & 141 & $-71(-108$ to -34$)$ & 239 & 151 & $-88(-127$ to -49$)$ & \\
\hline Idaho & 224 & 409 & 185 (136-234) & 267 & 485 & $218(164-272)$ & \\
\hline Illinois & 1,815 & 2,740 & $925(793-1,057)$ & 2,010 & 2,891 & $881(744-1,018)$ & \\
\hline Indiana & 954 & 2,154 & $1,200(1,091-1,309)$ & 1,063 & 2,389 & $1,326(1,211-1,441)$ & \\
\hline lowa & 485 & 859 & $374(302-446)$ & 528 & 968 & $440(364-516)$ & \\
\hline Kansas & 414 & 826 & $412(343-481)$ & 455 & 938 & $483(410-556)$ & \\
\hline Kentucky & 675 & 1,792 & $1,117(1,020-1,214)$ & 750 & 2,078 & $1,328(1,224-1,432)$ & * \\
\hline Louisiana & 658 & 1,106 & $448(366-530)$ & 733 & 1,360 & $627(537-717)$ & $*$ \\
\hline Maine & 237 & 443 & $206(155-257)$ & 268 & 494 & $226(172-280)$ & \\
\hline Maryland & 818 & 1,035 & $217(133-301)$ & 936 & 998 & $62(-24-148)$ & \\
\hline Massachusetts & 984 & 1,115 & $131(41-221)$ & 1,105 & 1,205 & $100(6-194)$ & \\
\hline Michigan & 1,527 & 2,721 & $1,194(1,066-1,322)$ & 1,712 & 2,939 & $1,227(1,093-1,361)$ & \\
\hline Minnesota & 762 & 960 & $198(117-279)$ & 871 & 1,153 & $282(194-370)$ & \\
\hline Mississippi & 446 & 1,016 & $570(495-645)$ & 492 & 1,129 & $637(558-716)$ & \\
\hline Missouri & 941 & 2,090 & $1,149(1,041-1,257)$ & 1,039 & 2,175 & $1,136(1025-1,247)$ & \\
\hline
\end{tabular}

See table footnotes on page 1253. 
TABLE 2. (Continued) Number of expected, observed, and potentially preventable deaths among the five leading causes of death and significant changes in potentially preventable deaths, for persons aged <80 years, by state — United States, 2010 and 2014

\begin{tabular}{|c|c|c|c|c|c|c|c|}
\hline \multirow[b]{2}{*}{ State } & \multicolumn{3}{|r|}{2010} & \multicolumn{3}{|r|}{2014} & \multirow{2}{*}{$\begin{array}{c}\text { Z-test } \\
\text { significance }\end{array}$} \\
\hline & Expected & Observed & Potentially preventable $(95 \% \mathrm{Cl})$ & Expected & Observed & Potentially preventable $(95 \% \mathrm{Cl})$ & \\
\hline Montana & 166 & 341 & $175(131-219)$ & 192 & 380 & $188(141-235)$ & \\
\hline Nebraska & 270 & 543 & $273(217-329)$ & 296 & 563 & $267(210-324)$ & \\
\hline Nevada & 395 & 701 & $306(241-371)$ & 472 & 883 & $411(339-483)$ & \\
\hline New Hampshire & 206 & 315 & 109 (64-154) & 237 & 352 & $115(67-163)$ & \\
\hline New Jersey & 1,312 & 1,436 & $124(21-227)$ & 1,447 & 1,391 & $-56(-160-48)$ & \\
\hline New Mexico & 320 & 535 & $215(158-272)$ & 361 & 605 & $244(183-305)$ & \\
\hline New York & 2,906 & 3,358 & $452(297-607)$ & 3,186 & 3,306 & $120(-38-278)$ & * \\
\hline North Carolina & 1,436 & 2,698 & $1,262(1,136-1,388)$ & 1,663 & 3,077 & $1,414(1,279-1,549)$ & \\
\hline North Dakota & 104 & 170 & $66(34-98)$ & 113 & 162 & $49(17-81)$ & \\
\hline Ohio & 1,818 & 3,729 & $1,911(1,765-2,057)$ & 1,996 & 3,922 & $1,926(1,775-2,077)$ & \\
\hline Oklahoma & 581 & 1,736 & $1,155(1,061-1,249)$ & 638 & 1,787 & $1,149(1,052-1,246)$ & \\
\hline Oregon & 599 & 1,110 & $511(430-592)$ & 706 & 1,153 & $447(363-531)$ & \\
\hline Pennsylvania & 2,101 & 3,051 & $950(809-1,091)$ & 2,287 & 3,223 & $936(791-1,081)$ & \\
\hline Rhode Island & 160 & 225 & $65(27-103)$ & 176 & 242 & $66(26-106)$ & \\
\hline South Carolina & 740 & 1,391 & $651(561-741)$ & 870 & 1,693 & $823(724-922)$ & \\
\hline South Dakota & 126 & 226 & $100(63-137)$ & 140 & 202 & $62(26-98)$ & \\
\hline Tennessee & 995 & 2,197 & $1,202(1,091-1,313)$ & 1,125 & 2,567 & $1,442(1,323-1,561)$ & * \\
\hline Texas & 3,139 & 5,061 & $1,922(1,745-2,099)$ & 3,656 & 5,456 & $1,800(1,613-1,987)$ & \\
\hline Utah & 298 & 383 & $85(34-136)$ & 350 & 451 & $101(46-156)$ & \\
\hline Vermont & 103 & 167 & $64(32-96)$ & 118 & 189 & 71 (37-105) & \\
\hline Virginia & 1,148 & 1,647 & $499(395-603)$ & 1,320 & 1,714 & $394(286-502)$ & \\
\hline Washington & 956 & 1,451 & $495(399-591)$ & 1,130 & 1,603 & $473(371-575)$ & \\
\hline West Virginia & 338 & 921 & $583(513-653)$ & 367 & 995 & $628(556-700)$ & \\
\hline Wisconsin & 862 & 1,190 & $328(239-417)$ & 970 & 1,375 & $405(310-500)$ & \\
\hline Wyoming & 83 & 186 & $103(71-135)$ & 95 & 185 & $90(57-123)$ & \\
\hline United States & 45,738 & 74,458 & $28,831(28,151-29,511)$ & 51,840 & 80,899 & $29,232(28,518-29,946)$ & \\
\hline \multicolumn{8}{|c|}{ Unintentional injuries (accidents) } \\
\hline Alabama & 910 & 2,036 & $1,126(1,020-1,232)$ & 939 & 2,104 & $1,165(1,057-1,273)$ & \\
\hline Alaska & 131 & 331 & $200(158-242)$ & 137 & 348 & $211(168-254)$ & \\
\hline Arizona & 1,191 & 2,341 & $1,150(1,034-1,266)$ & 1,284 & 2,562 & $1,278(1,156-1,400)$ & \\
\hline Arkansas & 551 & 1,221 & $670(587-753)$ & 568 & 1,172 & $604(522-686)$ & \\
\hline California & 6,886 & 8,627 & $1,741(1,497-1,985)$ & 7,315 & 9,818 & $2,503(2,246-2,760)$ & * \\
\hline Colorado & 940 & 1,525 & $585(488-682)$ & 1,018 & 1,833 & $815(710-920)$ & * \\
\hline Connecticut & 679 & 905 & $226(148-304)$ & 696 & 1,142 & $446(362-530)$ & * \\
\hline Delaware & 172 & 296 & $124(82-166)$ & 183 & 350 & $167(122-212)$ & \\
\hline District of Columbia & 117 & 169 & $52(19-85)$ & 128 & 177 & $49(15-83)$ & \\
\hline Florida & 3,675 & 6,927 & $3,252(3,050-3,454)$ & 3,951 & 6,997 & $3,046(2,841-3,251)$ & \\
\hline Georgia & 1,791 & 3,133 & $1,342(1,204-1,480)$ & 1,905 & 3,342 & $1,437(1,295-1,579)$ & \\
\hline Hawaii & 259 & 344 & $85(37-133)$ & 272 & 356 & $84(35-133)$ & \\
\hline Idaho & 285 & 516 & $231(176-286)$ & 304 & 575 & $271(213-329)$ & \\
\hline Illinois & 2,395 & 3,093 & $698(553-843)$ & 2,449 & 3,642 & $1,193(1,040-1,346)$ & * \\
\hline Indiana & 1,209 & 2,064 & $855(743-967)$ & 1,250 & 2,425 & $1,175(1,056-1,294)$ & * \\
\hline lowa & 571 & 892 & $321(246-396)$ & 587 & 948 & $361(284-438)$ & \\
\hline Kansas & 525 & 1,010 & $485(408-562)$ & 539 & 1,004 & $465(388-542)$ & \\
\hline Kentucky & 826 & 2,240 & $1,414(1,305-1,523)$ & 852 & 2,225 & $1,373(1,264-1,482)$ & \\
\hline Louisiana & 850 & 1,771 & $921(821-1,021)$ & 882 & 2,074 & 1,192 (1,085-1,299) & * \\
\hline Maine & 262 & 390 & $128(78-178)$ & 267 & 487 & $220(166-274)$ & \\
\hline Maryland & 1,093 & 1,065 & $-28(-119-63)$ & 1,147 & 1,217 & $70(-25-165)$ & \\
\hline Massachusetts & 1,252 & 1,507 & $255(152-358)$ & 1,310 & 2,085 & $775(661-889)$ & * \\
\hline Michigan & 1,869 & 2,923 & $1,054(918-1,190)$ & 1,916 & 3,455 & $1,539(1,395-1,683)$ & * \\
\hline Minnesota & 993 & 1,342 & 349 (254-444) & 1,034 & 1,440 & $406(309-503)$ & \\
\hline Mississippi & 553 & 1,395 & $842(756-928)$ & 567 & 1,438 & 871 (783-959) & \\
\hline Missouri & 1,133 & 2,328 & $1,195(1,080-1,310)$ & 1,164 & 2,414 & $1,250(1,133-1,367)$ & \\
\hline Montana & 190 & 416 & $226(178-274)$ & 199 & 418 & $219(170-268)$ & \\
\hline Nebraska & 337 & 490 & 153 (97-209) & 349 & 535 & $186(128-244)$ & \\
\hline Nevada & 510 & 952 & $442(367-517)$ & 549 & 1,032 & $483(405-561)$ & \\
\hline New Hampshire & 255 & 381 & $126(77-175)$ & 263 & 507 & $244(190-298)$ & * \\
\hline New Jersey & 1,665 & 1,888 & $223(106-340)$ & 1,718 & 2,309 & 591 (467-715) & * \\
\hline New Mexico & 386 & 1,013 & $627(554-700)$ & 397 & 1,249 & 852 (772-932) & * \\
\hline New York & 3,692 & 3,804 & $112(-58-282)$ & 3,813 & 4,515 & $702(523-881)$ & * \\
\hline North Carolina & 1,802 & 3,268 & $1,466(1,326-1,606)$ & 1,915 & 3,592 & $1,677(1,532-1,822)$ & \\
\hline North Dakota & 127 & 193 & $66(31-101)$ & 138 & 233 & $95(57-133)$ & \\
\hline
\end{tabular}

See table footnotes on the next page. 
Morbidity and Mortality Weekly Report

TABLE 2. (Continued) Number of expected, observed, and potentially preventable deaths among the five leading causes of death and significant changes in potentially preventable deaths, for persons aged <80 years, by state - United States, 2010 and 2014

\begin{tabular}{|c|c|c|c|c|c|c|c|}
\hline \multirow[b]{2}{*}{ State } & \multicolumn{3}{|r|}{2010} & \multicolumn{3}{|r|}{2014} & \multirow{2}{*}{$\begin{array}{c}\text { Z-test } \\
\text { significance }\end{array}$} \\
\hline & Expected & Observed & Potentially preventable $(95 \% \mathrm{Cl})$ & Expected & Observed & Potentially preventable $(95 \% \mathrm{Cl})$ & \\
\hline Ohio & 2,184 & 4,016 & $1,832(1,678-1,986)$ & 2,230 & 4,928 & $2,698(2,532-2,864)$ & * \\
\hline Oklahoma & 703 & 1,870 & $1,167(1,068-1,266)$ & 732 & 1,944 & $1,212(1,111-1,313)$ & \\
\hline Oregon & 730 & 1,068 & $338(255-421)$ & 773 & 1,254 & $481(393-569)$ & \\
\hline Pennsylvania & 2,435 & 4,319 & $1,884(1,723-2,045)$ & 2,486 & 4,993 & $2,507(2,337-2,677)$ & * \\
\hline Rhode Island & 200 & 339 & $139(93-185)$ & 205 & 408 & $203(154-252)$ & \\
\hline South Carolina & 883 & 1,910 & $1,027(923-1,131)$ & 942 & 2,032 & $1,090(983-1,197)$ & \\
\hline South Dakota & 151 & 284 & $133(92-174)$ & 159 & 320 & $161(118-204)$ & \\
\hline Tennessee & 1,209 & 2,895 & $1,686(1,560-1,812)$ & 1,268 & 3,059 & $1,791(1,662-1,920)$ & \\
\hline Texas & 4,551 & 7,612 & $3,061(2,845-3,277)$ & 4,951 & 8,159 & $3,208(2,984-3,432)$ & \\
\hline Utah & 470 & 765 & $295(226-364)$ & 510 & 927 & $417(343-491)$ & \\
\hline Vermont & 122 & 181 & $59(25-93)$ & 125 & 188 & $63(28-98)$ & \\
\hline Virginia & 1,521 & 1,889 & 368 (254-482) & 1,604 & 2,390 & $786(662-910)$ & * \\
\hline Washington & 1,269 & 1,925 & $656(545-767)$ & 1,355 & 2,181 & $826(709-943)$ & \\
\hline West Virginia & 364 & 1,031 & $667(594-740)$ & 368 & 1,134 & $766(690-842)$ & \\
\hline Wisconsin & 1,074 & 1,666 & $592(489-695)$ & 1,105 & 2,008 & $903(794-1,012)$ & * \\
\hline Wyoming & 106 & 296 & $190(151-229)$ & 111 & 315 & $204(164-244)$ & \\
\hline United States & 58,055 & 94,862 & $36,836(36,070-37,602)$ & 60,929 & 106,260 & $45,331(44,530-46,132)$ & * \\
\hline
\end{tabular}

Abbreviation: $\mathrm{Cl}=$ confidence interval.

* Significant change from 2010 to $2014, \mathrm{p}<0.01$.

${ }^{+}$Negative potentially preventable deaths occurred when a U.S. Department of Health and Human Services region included one or more of the states with the lowest three death rates (the lowest three death rates were averaged to create the benchmark death rates) for at least a few age groups. Negative potentially preventable deaths were preserved in this table to test changes from 2010 to 2014, but were truncated to zero and not included in the totals for the United States in the table and text.

increased for each of the five leading causes of deaths in 2014, and age-adjusted death rates declined during 2010-2014 for each category except unintentional injuries. Specifically, from 2010 to 2014 , age-adjusted death rates per 100,000 population for heart disease declined $6.8 \%$ from 179.1 to 167.0 ; for cancer, from 172.8 to 161.2 (6.7\% decrease); for stroke, from 39.1 to 36.5 (6.6\% decrease); and for CLRD, from 42.2 to 40.5 (4.0\% decrease). For unintentional injuries, age-adjusted death rates increased $6.6 \%$, from 38.0 to 40.5 (supplemental material at https://stacks.cdc.gov/view/cdc/42341) (1). Among subcategories of unintentional injury deaths for all ages, ageadjusted death rates for poisonings increased 25\%, and falls increased by $12 \%$ (supplemental material at https://stacks. cdc.gov/view/cdc/42344). Prescription drug and illicit drug overdose was a major contributor to the increase in poisonings during 2010-2014 (4).

\section{Discussion}

The results of this analysis show that the number of observed deaths increased for each of the leading five causes of death, consistent with increases in population size in 2014, compared with 2010. Age-adjusted death rates declined overall for all causes of death combined in 2014 compared with 2010. Potentially preventable deaths declined during 2010-2014 for three of the five leading causes of death: diseases of the heart, cancer, and stroke. No change was observed for potentially preventable deaths from CLRD. Potentially preventable deaths from unintentional injuries increased from 2010 compared with 2014.
States in the Southeast continued to have the highest number of potentially preventable deaths from all five causes in 2014.

Although substantial progress was made in combatting infectious diseases during the early part of the 20th century, additional focus has shifted toward prevention of noncommunicable diseases, including chronic diseases, and unintentional injuries $(5,6)$. The decrease in cancer deaths can be attributed, in part, to progress in prevention, early detection, and treatment ( 7 ). Improvement of quality of care and reduction in risk factors, including increased number of persons with hypertension under control, have contributed to the decline in death rates for heart disease and stroke. ${ }^{* *}$ Tobacco use is a risk factor for some of the deaths included in this report, such as heart disease, cancer, CLRD, and cerebrovascular diseases. ${ }^{\dagger \dagger}$ Mortality from tobacco-related causes has decreased in conjunction with national decreases in tobacco use across the United States, but an estimated 40 million adults (16.8\%) smoked in 2014 (8). Implementation of evidence-based tobacco control interventions, including increased tobacco product prices, implementation and enforcement of comprehensive smoke-free laws, media campaigns, and access to proven resources (e.g., quit lines) to help persons quit tobacco use $\mathrm{S}^{\mathbb{S}}$ varies among states. In addition to tobacco use, other health behaviors contribute to premature deaths and create opportunities for prevention. For example,

\footnotetext{
** http://www.cdc.gov/nchs/data/databriefs/db220.pdf.

${ }^{\dagger \dagger}$ http://www.surgeongeneral.gov/library/reports/50-years-of-progress/.

$\$ \$$ http://www.cdc.gov/tobacco/stateandcommunity/best_practices/index.htm.
} 
obesity increases the risk for CLRD, diseases of the heart, and cerebrovascular disease, in addition to some cancers. 99

Although the number of potentially preventable deaths declined during 2010-2014 for heart disease, cancer, and stroke, observed deaths increased overall for these causes. Based on the methodology used for this analysis, when the pace of the increase in observed deaths is slower than the growth in population, potentially preventable deaths will decrease. Observed deaths increased 6\% for heart disease, $4 \%$ for cancer, $4 \%$ for stroke, and $8 \%$ for CLRD. These increases were smaller than would be expected to result from population growth, particularly growth in population size among older age groups during this period.

In contrast, both observed and potentially preventable deaths from unintentional injuries increased during 2010-2014. Examples of state actions to reduce drug overdose include developing or enhancing prescription drug monitoring programs, adopting clinical prescribing guidelines, and increasing access to medication-assisted treatment for opioid use disorder and naloxone to reverse opioid-related poisoning (9). As the U.S. population aged, falls among older adults increased. Tools such as STEADI, designed to assist clinicians in assessing fall risk, educating patients, and selecting interventions, are available from CDC.***

The findings in this report are subject to at least five limitations. First, the same method used in a previous report was applied to set a benchmark for potentially preventable deaths (2). These benchmarks are based on data from the states with the lowest death rates for each condition during 2008-2010 alone. The benchmarks might need to be reevaluated over time, especially given shifts in cause-specific death rates observed

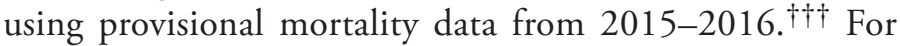
example, death rates from unintentional injury were increasing before 2008-2010, resulting in benchmarks that might not be comparable to historical lows or international points of reference. Second, alternative ways of defining and measuring potentially preventable or premature avoidable mortality have been used in other studies and no gold standard exists (10). Third, a lowest average rate was calculated based on individual states. The sum of the individual potentially preventable deaths by state is qualitatively different from estimating the number of potentially preventable deaths for the United States as a whole. Fourth, changes in the number of potentially preventable deaths by cause are not necessarily independent. For example, whereas some cancer deaths might be prevented entirely, some might be shifted into another cause grouping, such as heart

\footnotetext{
99 http://www.nhlbi.nih.gov/sites/www.nhlbi.nih.gov/files/obesity-evidencereview.pdf.

*** http://www.cdc.gov/steadi/pdf/stay_independent_brochure-a.pdf.

$\dagger_{\dagger}{ }^{\dagger}$ http://www.cdc.gov/nchs/products/vsrr/mortality-dashboard.htm.
}

\section{Summary \\ What is already known about this topic?}

Deaths from heart disease, cancer, chronic lower respiratory disease, cerebrovascular diseases (stroke), and unintentional injuries account for the five leading causes of death in the United States. Death rates for these diseases vary widely across states, related to variation in the distribution of social determinants of health, access and use of health services, and public health efforts.

What is added by this report?

There has been a significant decrease in the number of potentially preventable deaths among three of the five leading causes of death (diseases of the heart, cancer, and stroke) during 2010-2014. However, the number of potentially preventable deaths from unintentional injuries increased significantly during the same period. This is mostly attributed to an increase in drug poisoning (overdose from prescription and illicit drugs) and falls. No significant change was observed in potentially preventable deaths from chronic lower respiratory disease (e.g., asthma, bronchitis, and emphysema).

What are the implications for public health practice?

Public health officials can use the decreases observed as benchmarks for improving population health, while using observed increases to direct targeted efforts to reduce the number of potentially preventable deaths. A joint effort of public health and health care organizations can support analysis and action to reduce the number of potentially preventable deaths from the five leading causes of death. Specifically, given the reported increase in potentially preventable deaths from unintentional injuries, these findings might inform the selection and implementation of evidencebased interventions to prevent deaths from injuries such as falls and drug overdoses, based on epidemiologic burden.

disease. Finally, defining potentially preventable deaths across the five leading causes does not take into consideration the fact that these are complex and diverse causes of death. Not all deaths are equivalently preventable across the leading causes or within each leading cause. For example, certain types of cancer might be considered more or less preventable than other types, and some specific mechanisms of injury deaths (e.g., drug poisoning) might be considered completely preventable and other mechanisms less preventable. In addition, the majority of risk factors do not occur randomly in populations; they are closely related to the social, demographic, environmental, economic, and geographic attributes of the neighborhoods in which persons live and work. $\$ \$ \$$ However, from a health equity perspective, every state can be compared with the same benchmark rates regardless of demographic differences. If health disparities were eliminated, as is called for by Healthy People 2020,999 all

\footnotetext{
$\$ \$ \$$ http://www.cdc.gov/mmwr/preview/ind2013_su.html. 999 https://www.healthypeople.gov/.
} 
states could be closer to achieving the lowest possible death rates for the five leading causes of death.

Further analysis of state and regional differences in death rates for the five leading causes of death could assist state and federal health officials in establishing prevention goals, priorities, and strategies. Clinical preventive services, including physician tobacco cessation counseling, as recommended by the U.S. Preventive Services Task Force**** for heart disease, stroke, cancer, and CLRD also provide opportunities for addressing preventable deaths. ${ }^{\dagger \dagger \dagger}$

\footnotetext{
**** https://www.uspreventiveservicestaskforce.org/Page/Name/ uspstf-a-and-b-recommendations/.

$i^{\dagger}+\dagger$ https://www.uspreventiveservicestaskforce.org/Page/Name/ tools-and-resources-for-better-preventive-care.
}

\section{Acknowledgments}

Chad Heilig, PhD, Paul Z. Siegel, MD, Center for Surveillance, Epidemiology, and Laboratory Services, CDC; Peter Briss, MD, National Center for Chronic Disease Prevention and Health Promotion, CDC.

\footnotetext{
${ }^{1}$ Center for Surveillance, Epidemiology, and Laboratory Services, CDC; ${ }^{2}$ National Center for Health Statistics, CDC; ${ }^{3}$ National Center for Injury Prevention and Control, CDC; ${ }^{4}$ National Center for Chronic Disease Prevention and Health Promotion, CDC.

Corresponding author: Macarena C. García, mcgarcia@cdc.gov, 404-539-4410.
}

\section{References}

1. Kochanek KD, Murphy SL, Xu J, Tejada-Vera B. Deaths: final data for 2014. Natl Vital Stat Rep 2016;65(4):1-122.

2. Yoon PW, Bastian B, Anderson RN, Collins JL, Jaffe HW. Potentially preventable deaths from the five leading causes of death-United States, 2008-2010. MMWR Morb Mortal Wkly Rep 2014;63:369-74.

3. Heron M. Deaths: leading causes for 2014. Natl Vital Stat Rep 2016;65(5):1-96.

4. Rudd RA, Aleshire N, Zibbell JE, Gladden RM. Increases in drug and opioid overdose deaths-United States, 2000-2014. MMWR Morb Mortal Wkly Rep 2016;64:1378-82. http://dx.doi.org/10.15585/ mmwr.mm6450a3

5. Epidemiology Program Office, Office of the Director, CDC. Achievements in public health, 1900-1999: changes in the public health system. MMWR Morb Mortal Wkly Rep 1999;48:1141-7.

6. Bauer UE, Briss PA, Goodman RA, Bowman BA. Prevention of chronic disease in the 21st century: elimination of the leading preventable causes of premature death and disability in the USA. Lancet 2014;384:45-52. http://dx.doi.org/10.1016/S0140-6736(14)60648-6

7. Ryerson AB, Eheman CR, Altekruse SF, et al. Annual report to the nation on the status of cancer, 1975-2012, featuring the increasing incidence of liver cancer. Cancer 2016;122:1312-37. http://dx.doi.org/10.1002/cncr.29936

8. Jamal A, Homa DM, O'Connor E, et al. Current cigarette smoking among adults_-United States, 2005-2014. MMWR Morb Mortal Wkly Rep 2015;64:1233-40. http://dx.doi.org/10.15585/mmwr.mm6444a2

9. Haegerich TM, Paulozzi LJ, Manns BJ, Jones CM. What we know, and don't know, about the impact of state policy and systems-level interventions on prescription drug overdose. Drug Alcohol Depend 2014;145:34-47. http://dx.doi.org/10.1016/j.drugalcdep.2014.10.001

10. Schieb LJ, Greer SA, Ritchey MD, George MG, Casper ML. Vital signs: avoidable deaths from heart disease, stroke, and hypertensive disease - United States, 2001-2010. MMWR Morb Mortal Wkly Rep 2013;62:721-7. 